\title{
The Effect of Flexibility of Bridge and Plank Exercises using Sling Suspension on an Unstable Surface on while Standing in Healthy Young Adults Kyung-Tae Yoo, PT, $\mathrm{PhD}^{\dagger}$ \\ Department of Physical Therapy, Namseoul University
}

Received: March 11, 2016 / Revised: March 14, 2016 / Accepted: April 13, 2016

(c) 2016 J Korean Soc Phys Med

\section{| Abstract |}

PURPOSE: The purpose of this study was to identify the effects on flexibility of bridge and plank exercises using sling suspension on an unstable surface.

METHODS: The subjects of this study were 20 healthy adults in their 20s (plank $=10$, bridge $=10$ ). Both types of exercise were performed three times per week for a period of four weeks. Each exercise was performed in the front and side direction. Exercise intensity was altered through the use of a sling, which was placed at the knee and ankle. Flexibility at trunk forward flexion and backward extension was measured. The trunk forward flexion was measured at sitting position. The trunk backward extension was measured at prone position. The data were analyzed by Two-way ANOVA.

RESULTS: There were significant differences in the preand post-test for both the bridge and plank exercise groups. In the bridge exercise, significant differences were shown in the trunk forward flexion and the trunk backward extension $(\mathrm{p}<.05)$. In the plank exercise, a significant difference was shown in the trunk backward extension $(p<.05)$. No

†Corresponding Author : taeyoo88@nsu.ac.kr

This is an Open Access article distributed under the terms of the Creative Commons Attribution Non-Commercial License (http://creativecommons.org/licenses/by-nc/3.0) which permits unrestricted non-commercial use, distribution, and reproduction in any medium, provided the original work is properly cited. significant differences were noted in interaction effect or the main effects in either group.

CONCLUSION: Bridge and plank exercises on an unstable surface improve flexibility. The bridge exercise improves the flexibility of the forward and backward muscles of the trunk. The plank exercise improves the flexibility of the forward muscles of the trunk. This information would be useful in the development of exercise programs including bridge and plank exercises for improving flexibility and core stability.

Key Words: Bridge exercise, Flexibility, Plank exercise, Sling

\section{Introduction}

Prolonged periods of computer work and the frequent use of smart phones have resulted in escalating instances of poor posture. There is a general lack of awareness about this condition (Lee and Kim, 2014). Core stability training (CST) is being emphasized in many studies to counteract health problems resulting from sedentary lifestyles and bad posture. CST has been shown to have positive effects on both patients and athletes (Miyake et al., 2013).

The core is the central part of the body and comprises 
a complex made up of the lumbar vertebrae, pelvis, and hip joint. The core is fixed by the trunk and abdominal muscles, which maintain the stability of the spine (Miyake et al., 2013). The stabilization of the core is enabled by continuous adjustment of muscular tension by the central nervous system. The muscles involved in the stabilization of the lower trunk include the transversus abdominis muscle, obliquus internus abdominis muscle, and external obliquus externus abdominis muscle, which are located in the front, and the paravertebral muscle, multifidus muscle, diaphragm, pelvic floor muscle, and gluteus muscle, which are located in the back around the hip joint. The global muscle is a lamellar muscle in the abdomen and back that is used as the agonistic muscle for trunk movement; the local muscle is in the deep part of the abdominal walls and is involved in posture control, which is related to the stability of the lumbar spine (Marshall and Murphy, 2005).

The bridge exercise is a core stability exercise. It is a closed chain exercise in which a knee-standing posture is performed while bearing weight. This exercise develops the muscles involved in the control of standing from a sitting position and reinforces the lower spine and hip extensors for preparing for the stance phase of the walking motion (O'Sullivan and Schmitz, 2001). This bridge exercise focuses on retraining the muscle coordination pattern at a proper ratio between the segmental stabilization of fine motor and the generation of gross motor torque. It is also used as a method to improve trunk stabilization at the pelvic area and has been applied to increase hip extensor and hamstring strength (Stevenson et al., 2007).

The plank exercise is a representative core stability exercise that increases the activity of trunk muscles (Ekstrom et al., 2007). It has been proven more effective to activate the global and local muscles together rather than training one joint alone (Marshall and Murphy, 2005), and increasing the load through limb movement increases the activity of the core muscles (Kim et al., 2013).

A sling can be used during exercise to either remove the effect of gravity and offers patients an active exercise therapy (Kim and Kwon, 2001). Sling exercise contributes to lumbar stabilization and can provide muscle stabilizing effects by improving joint mobility, stretching, muscle endurance, and relaxation (Lee et al., 2015).

In a previous study, the study was to investigate the flexibility on sling exercises for 6 weeks on women in their 20's with back pain. The study resulted that there were significant differences in the sling exercise group (Seo et al., 2013). A study on fin swimmers that performed core muscle strengthening training for 12 weeks, muscle activity significantly increased in trunk backward extension (Lee et al., 2012). When the effects of bridge exercise were analyzed through trunk core muscle activity according to the sling height in hip joint abduction and adduction in healthy adults, a high sling height was most effective for increasing trunk muscle activity (Lee et al., 2015). A previous study on healthy adults conducted core stability exercises with and without a Swiss ball and analyzed EMG changes. The researchers reported that muscle activity significantly increased in the rectus abdominis when a single-leg position was maintained using a Swiss ball (Marshall and Murphy, 2005). In another study on healthy adults, bridge exercises were performed on stable and unstable surfaces and the muscle activities of the trunk and lower limbs were analyzed. It was reported that the muscle activity increased more on the unstable surface than on the stable surface (Lee et al., 2010). Furthermore, a study was to investigate the effects of trunk stabilization exercise using a Swiss ball and core stabilization exercise on balance and gait in elderly women who had limitations in balance and gait. The result of the study was that trunk stabilization exercise using a Swiss ball improved balance and gait better than core stabilization exercise (Choi et al., 2012). Many studies have been conducted on core stability exercises, but most of these studies provided instability using a Swiss ball rather than investigating muscle variables, such as the muscle activity of trunk muscles. And the studies on comparisons between stable and unstable surfaces referred that unstable surface was 
more effective to the muscle activity. Also, in the previous studies on flexibility, one exercise group such as sling exercise or core stabilize exercise including bridge and plank exercises respectively or combining core activity exercises with other exercise methods. The flexibility alters according to the muscle variables and the unstable surface caused different muscle activity compared to the stable surface Therefore, this study examined the effects on flexibility of bridge and plank exercises, which are core stability exercises, using slings on an unstable surface and would help to further studies related to flexibility and core stability exercises. This study hypothesized that (1) there would be significant differences between the groups in the bridge exercise group and plank exercise group when on stable and unstable surface. (2) there would be significant differences between pre- and post-exercises in the bridge exercise group and plank exercise group when on stable and unstable surface.

\section{Method}

\section{Subjects}

The subjects of this study were 20 healthy men and women in their 20s. Those who had musculoskeletal diseases, nervous system diseases, athletic careers, and flexibility training experience during the previous six months were excluded. The purpose and content of this experiment were explained to the subjects and once these concepts were understood, the subjects voluntarily signed consent forms before participating. Twenty healthy adults were selected for this study and randomized to two groups of 10 members respectively. The average age, height, weight, and body mass index (BMI) of the bridge exercise group were $22.40 \pm 1.84$ years, $167.06 \pm 10.18 \mathrm{~cm}, 63.70 \pm 13.32 \mathrm{~kg}$, and $22.58 \pm 2.83 \mathrm{~kg} / \mathrm{m}^{2}$, respectively. The average age, height, weight, and BMI of the plank exercise group were $22.60 \pm 1.58$ years, $167.16 \pm 10.96 \mathrm{~cm}, 63.68 \pm 14.70 \mathrm{~kg}$, and $22.59 \pm 3.49 \mathrm{~kg} / \mathrm{m}^{2}$, respectively (Table 1).

Table 1. General characteristics of the subjects

$(n=20)$

\begin{tabular}{lll}
\hline Variables & $\begin{array}{c}\text { Bridge exercise } \\
(\mathrm{n}=10)\end{array}$ & $\begin{array}{c}\text { Plank exercise } \\
(\mathrm{n}=10)\end{array}$ \\
\hline Age (years) & $22.40 \pm 1.84$ & $22.60 \pm 1.58$ \\
Height $(\mathrm{cm})$ & $167.06 \pm 10.18$ & $167.16 \pm 10.96$ \\
Weight $(\mathrm{kg})$ & $63.70 \pm 13.32$ & $63.68 \pm 14.70$ \\
BMI $\left(\mathrm{kg} / \mathrm{m}^{2}\right)$ & $22.58 \pm 2.83$ & $22.59 \pm 3.49$ \\
\hline
\end{tabular}

Expressed as Mean \pm Standard deviation

\section{Measuring Equipment}

A body composition analyzer (InBody720, Biospace O. Ltd., Republic of Korea) was used to measure the physical characteristics of the subjects (Fig. 1), and the Redcord Professional Workstation (Sliding Suspension System w/ 3 Trainers, Redcord, Norway) was used for the sling to reduce the load during the bridge exercise (Fig. 2) and the plank exercise (Fig. 3). For trunk forward flexion, a trunk forward

Table 2. Flexibility comparisons according to group and time (cm)

\begin{tabular}{|c|c|c|c|c|c|}
\hline Variables & Group & Pre-test & Post-test & & \\
\hline \multirow[t]{3}{*}{ Trunk forward flexion } & Bridge exercise & $9.12 \pm 11.66$ & $13.60 \pm 10.42^{+}$ & group & .72 \\
\hline & \multirow[b]{2}{*}{ Plank exercise } & \multirow[b]{2}{*}{$11.09 \pm 8.79$} & \multirow[b]{2}{*}{$14.79 \pm 8.38^{+}$} & time & $.00 * * *$ \\
\hline & & & & group $*$ time & .54 \\
\hline \multirow{3}{*}{$\begin{array}{l}\text { Trunk backward } \\
\text { extension }\end{array}$} & \multirow{2}{*}{ Bridge exercise } & \multirow{2}{*}{$31.40 \pm 6.93$} & \multirow{2}{*}{$39.71 \pm 8.03^{+}$} & group & .25 \\
\hline & & & & time & $.00 * * *$ \\
\hline & Plank exercise & $29.47 \pm 8.60$ & $33.77 \pm 7.56$ & group $*$ time & .06 \\
\hline
\end{tabular}

Expressed as Mean \pm Standard deviation, ${ }^{* * *} \mathrm{p}<.001,{ }^{+}$significant difference between pre-test and post-test 


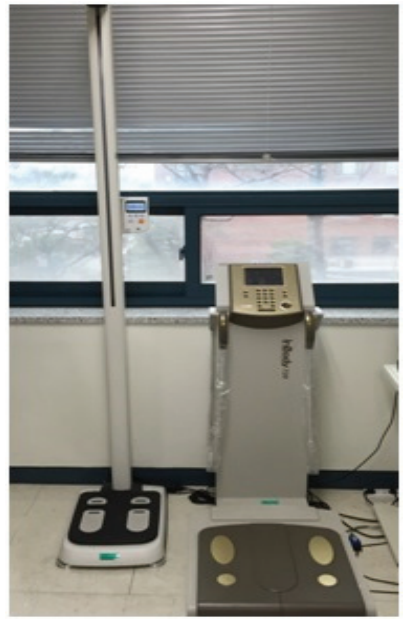

Fig. 1. Body composition analyzer (InBody720, Biospace O. Ltd., Republic of Korea)
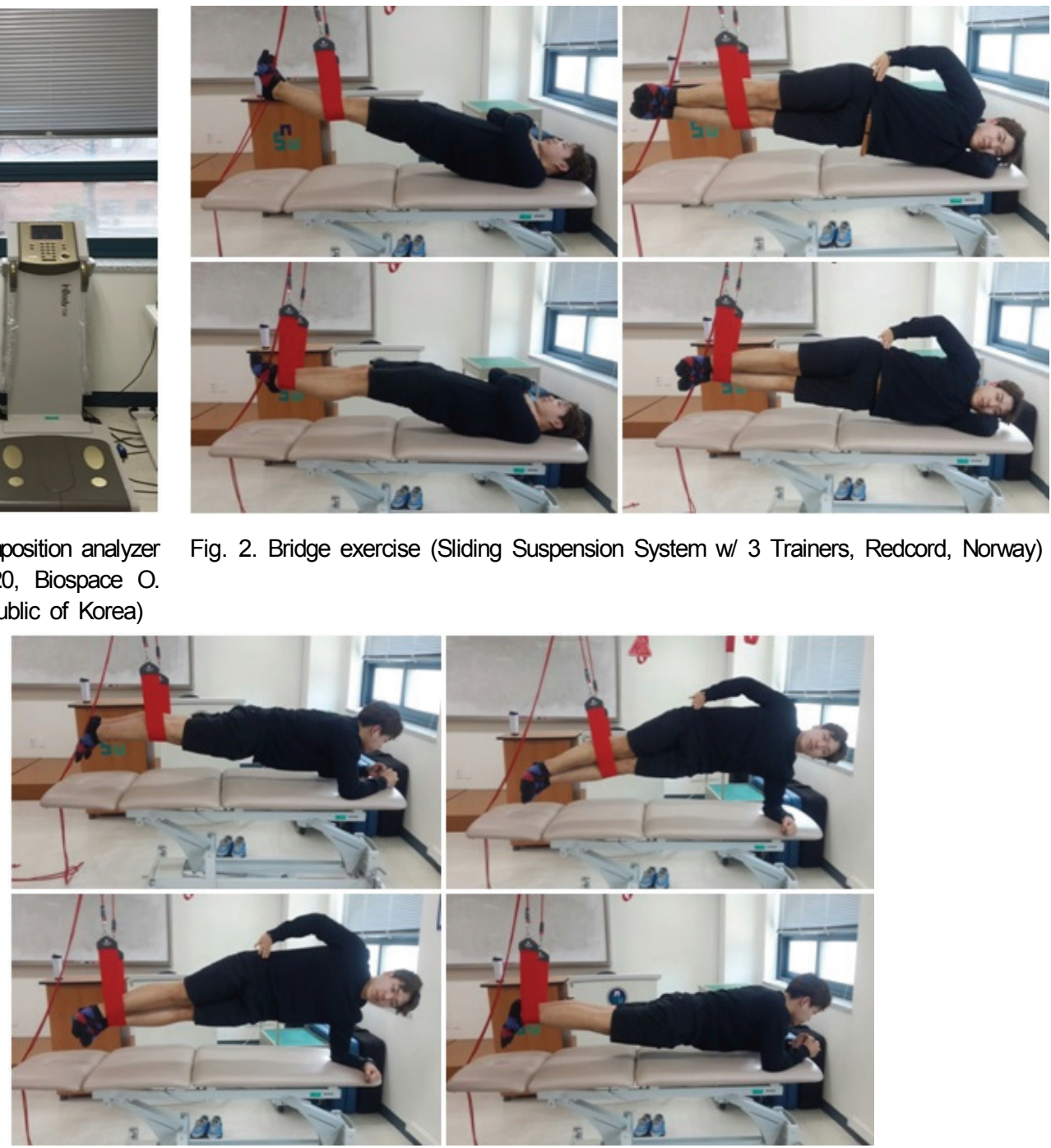

Fig. 2. Bridge exercise (Sliding Suspension System w/ 3 Trainers, Redcord, Norway)

Fig. 3. Plank exercise (Sliding Suspension System w/ 3 Trainers, Redcord, Norway)

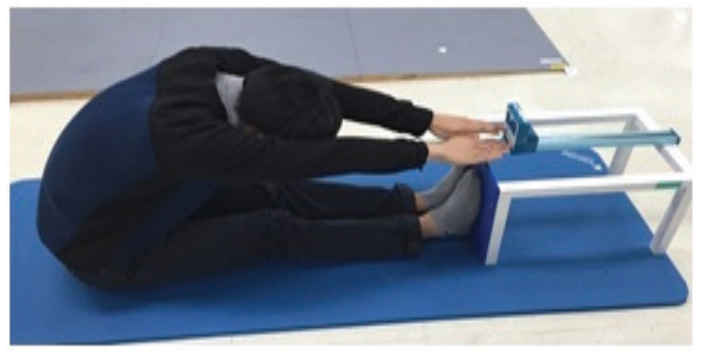

Fig. 4. Trunk forward flexion meter (DW-782 Flexion Meter, DSI, Republic of Korea)

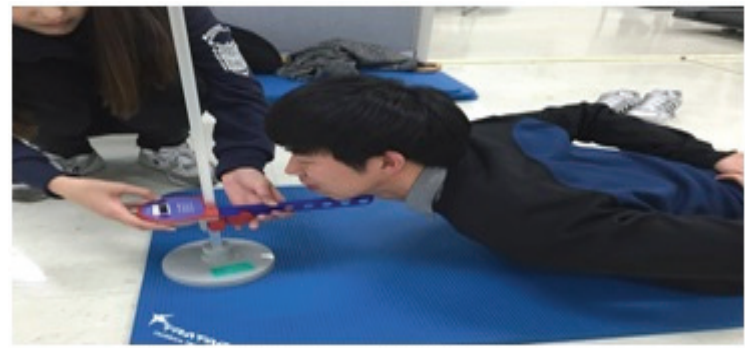

Fig. 5. Digital Backward Flexmeter (T.K.K. 5404 Extension-D, Takei Scientific Instruments Co. Ltd., Japan) 
flexion meter was used (DW-782 Flexion Meter, DSI, Republic of Korea), and for trunk backward extension (Fig. 4), the Digital Backward Flexmeter (T.K.K. 5404 Extension-D, Takei Scientific Instruments Co. Ltd., Japan) was used (Fig. 5).

\section{Procedures}

The 20 subjects were randomized to two groups; the bridge exercise group and the plank exercise group with 10 members in each group. The subjects did not know the effects of these exercise protocols. This study was performed as single-blinded in a laboratory. The exercise method of Lee (2012) was modified for this study and the modified exercises were performed three times a week for four weeks. The total exercise protocol time was 60 minutes, which consisted of warming up for 10 minutes, the main exercise for 40 minutes, and a cool-down exercise for 10 minutes. For the main exercise, front direction, left side direction, and right side direction exercises were performed, in this order, using a sling that was hung 50 $\mathrm{cm}$ above the mat (Lee, 2012) for the bridge exercise and the plank exercise, respectively. To increase exercise intensity, the sling was positioned at the knee joint in level 1 and at the ankle joint in level 2. For the front position of the bridge exercise, the subject raised the pelvis until the hip joint angle was $0^{\circ}$ in the crook-lying position where the subject lies down with the knees bent $90^{\circ}$ in the supine position (Czaprowski et al., 2014) and maintained for 10 seconds the position (Kim et al., 2014). For the front position of the plank exercise, the subject maintained a neutral position at the hip joints, pelvis and lumbar spine in the prone position and took the side plank position, supported by the left forearm. Then the right elbow was placed under the glenohumeral joints and the body was supported with the arms perpendicular to the surface (Czaprowski et al., 2014). For the side exercises, subjects lay on their sides with the shoulder height identical to that of the front position and maintained for 10 seconds the position. They performed isometric contraction, in which the end motion was stopped for 10 seconds (Lee, 2012), and then had one minute of resting time between the sets to prevent a fatigue effect (Czaprowski et al., 2014).

Flexibility was measured using the trunk forward flexion and backward extensions before and after the exercise. These methods are widely used to measure the flexibility of the trunk; trunk forward flexion (Ayala et al., 2012; Baltaci et al., 2003; Holt et al., 1999) and trunk backward extension (Kang, 2010; Park, 2009; Lee et al., 2009). For the trunk forward flexion, the subject sat on the mat with soles touching the equipment and knees extended. Then the upper body was bent forward to the maximum and both arms and hands were extended to push the measuring instrument as much as possible. This position was held for at least five seconds (Mika et al., 2013) and the point where the subject's fingertip touched was measured in .1 $\mathrm{cm}$ units. For the trunk backward extension, the subject lay face down with both hands held together behind the pelvic area. To prevent compensation, an assistant fixed the position by pressing on the subject's knees and back of the thighs. The subject raised the upper body including the chin and head as far as possible and maintained the position until measurement. Then the height of the chin from the baseline was measured in $.1 \mathrm{~cm}$ units. Both the trunk forward flexion and backward extensions were measured twice and the higher recorded value was adopted.

\section{Statistical analysis}

The statistics application SPSS version 20.0 for Windows was used for data processing in this study. For data characteristics, the normal distribution was proven through the kolmogorov-smirnov test and the homogeneity of subjects was verified using the Levene F-test. To analyze flexibility by group (bridge exercise and plank exercise) and time (pre- and post-test), the two-way ANOVA (analysis of variance) was used. The statistical significance level was set at $\alpha=.05$. 


\section{Results}

The comparison of flexibility by exercise method showed no interaction effects between group and time and no main effect of group. However, a significant difference between the times was found for both the trunk forward flexion and backward extension (Table 2). In the trunk forward flexion, significant differences between pre- and post-test were found for both the bridge and plank exercise groups, and in the trunk backward extension, a significant difference between pre- and post-test was found in the bridge exercise group.

\section{Discussion}

During the performance of movement, an unstable surface increases the activity of the core muscles and maintains stability more effectively than a stable surface (Vera-Garcia et al., 2000). In this study, the bridge and plank exercises were performed on an unstable surface using a sling during 4 weeks in the 20 healthy young adults. As the flexibility measurement method, in the trunk forward flexion, there were significant differences between pre- and post-test in both the bridge and plank exercise groups. In the trunk backward extension, there was a significant difference between pre- and post-test in the bridge exercise group.

In a previous study on adult women, modern Pilates mat exercises were performed in a sitting position; these exercises were found to have positive effects on abdominal and lower back muscular strength, abdominal muscular endurance, and posterior trunk flexibility (Sekendiz et al., 2007). A study was to analyze the Schroth exercise method and sling exercise method performed with scoliosis. These results revealed that two exercise program improved flexibility, static standing balance, spine angle, chest expansion level and ability but the sling exercise was more effective (Lee and Kim, 2014). A study was to identify the flexibility, trunk forward flexion and backward extension on sling exercises and control group for 6 weeks on young healthy. The study resulted that the sling exercise group showed significant differences in the trunk forward flexion and backward extension while the control group showed a significant difference in the trunk backward extension (Seo et al., 2013). A study which was related to relationship between flexibility and EMG activity pattern of erector spinae muscles during trunk flexion and extension reported that flexibility plays an important role in trunk muscular recruitment pattern (Hashemirad et al., 2009) EMGs of the abdominal muscles were measured on stable and unstable surfaces, and the highest activity was observed during the prone bridge exercise on a Swiss ball (Czaprowski et al., 2014). A study was to analyze the trunk muscle activity during bridge exercise with various shoulder support surface; stable, sling, and Swiss ball. The result was RA and EO was recorded the highest activity during the bridge exercise with their shoulder on a sling (Son, 2015). When the effects of complex hip movements on healthy adults using a sling in bridge exercises; bridge exercise, bridging with unilateral hip movement, and bridging with bilateral hip movement on the EMG of the trunk muscles were analyzed, bridging exercises with bilateral movement were the most useful in increasing the EMG activity of obliquus internus abdominis and multifidus muscles; this activity was associated with the global muscles during the bridge exercise (Park et al., 2014). Furthermore, analysis of the trunk and lower extremity muscle activity of healthy adult women during various bridge exercises on an unstable surface showed that the use of an unstable surface increased the muscle activity of the trunk and lower extremities; the single leg lift bridge exercise increased the activities of both obliquus externus abdominis muscles, the erecter spinae, and the biceps femoris (Kim et al., 2014).

The results of this study were similar to the previous 
results. In the present study, there was a significant difference in the trunk forward flexion between pre- and post-test for both groups and a significant difference in the trunk backward extension between pre- and post-test for the bridge exercise group. This is because the bridge and plank exercises were performed in the side directions as well as in the front direction, which increased not only the activity of the abdominal and back muscles, which are the main muscles for front and back movement, but also the activity of the obliquus internus abdominis muscle, which is the main muscle for side movement as well as the obliquus externus abdominis muscle that helps it, and this increased general flexibility. In particular, a significant difference was shown in the trunk forward flexion because the side muscles assist the forward bending motion.

No significant difference was shown in the trunk backward extension for the plank exercise because the trunk backward extension mostly requires strength in the back muscles. When the muscle activities of the trunk and lower extremities were analyzed after the bridge exercise on stable and unstable surfaces in a preceding study, a significant difference was shown in the muscle located in the dorsal part for the bridge exercise on an unstable surface (Roh and Ma, 2012). However, it must have been difficult to show a significant difference between the pre- and post-test with the plank exercise, which helps the development of upper muscles rather than low back muscles.

A limited number of subjects (20) were used in this study. Furthermore, it was difficult to make direct associations with EMG and muscle strength because only flexibility was measured, even though this was a study about the effectiveness of muscles. Therefore, in future research, additional variables need to be measured to obtain more detailed data.

\section{Conclusion}

In this study, healthy adults performed bridge and plank exercises using a sling on an unstable surface and the effects on flexibility were examined. No group interaction effects or main effects were found, but a significant difference between pre- and post-test was shown in the trunk forward flexion for both the bridge and plank exercises, and in the trunk backward extension for the bridge exercise group. This result suggests that bridge and plank exercises increase the activities of various core muscles and also increase flexibility. Therefore, existing exercise programs that focus on stretching to enhance flexibility could be improved to produce better results by including core stability exercises on an unstable surface. The findings of this study will be helpful for research on core stability exercises, including bridge exercises and plank exercises using a sling.

\section{Acknowledgment}

Funding for this research was provided by Namseoul university.

\section{References}

Ayala F, Sainz de Baranda P, De Ste Croix M, et al. Reproducibility and criterion-related validity of the sit and reach test and toe touch test for estimating hamstring flexibility in recreationally active young adults. Phys Ther Sport. 2012;13(4):219-26.

Baltaci G, Un N, Tunay V, et al. Comparison of three different sit and reach tests for measurement of hamstring flexibility in female university students. Br JSportsMed. 2003;37(1):59-61.

Choi SH, Lim JH, Cho HW, et al. The Effects of Trunk Stabilization Exercise Using Swiss Ball and Core Stabilization Exercise on Balance and Gait in Elderly Women. J Korean Soc Phys Med. 2012;7(1):49-58. Czaprowski D, Afeltowicz A, Gbicka A, et al. Abdominal muscle 
EMG-activity during bridge exercises on stable and unstable surfaces. Phys Ther Sport. 2014;15(3):162-8.

Ekstrom RA, Donatelli RA, Carp KC. Electromyographic analysis of core trunk, hip, and thingh muscles during 9 rehabilitation exercises. J Orthop Sports Phys Ther. 2007;37(12):743-62.

Hashemirad F, Talebian S, Hatef B, et al. The relationship between flexibility and EMG activity pattern of the erector spinae muscles during trunk flexion-extension. J Electromyogr Kinesiol. 2009;19(5):746-53.

Holt LE, Pelham TW, Burke DG. Modifications to the standard sit and-reach flexibility protocol. J Athl Train. 1999;34(1):43-7.

Kang SR, Jeong GY, Moon DA, et al. Characteristic Analysis of Flexibility and Muscle Strength according to Exercise using Lumbar Strengthen Exercise Instrument. Journal of Rehabilitation Welfare Engineering \& Assistive Technology. 2010;4(1):53-61.

Kim JH, Kim Y, Chung Y. The Influence of an Unstable Surface on Trunk and Lower Extremity Muscle Activities during Variable Bridging Exercises. J Phys Ther Sci. 2014;26(4):521-3.

Kim MJ, Oh DW, Park HJ. Integrating arm movement into bridge exercise: Effect on EMG activity of selected trunk muscles. J Electromyogr Kinesiol. 2013;23(5): 1119-23.

Kim SY, Kwon JH. Lumbar stabilization exercises using the sling system. J Korean Acad Orthop Man Phys Ther. 2001;7(2):23-40.

Lee DG, Ahn SH, Oh JK, et al. The Effects of Swiss Ball Lumbar Stabilization Exercise on the Strength and Flexibility, Balance. J Korean Acad Clin Electrophysiol. 2009;7(1):35-42.

Lee DH, Park JS, Lee SY. Effects of bridge exercise on trunk core muscle activity with respect to sling height and hip joint abduction and adduction. J Phys Ther Sci. 2015;27(6):1997-9.

Lee JH. Changes in Trunk Muscle Activity Resulting from
Differences in the Bearing Surface and Bridging Methods. Mastrer's Degree. Catholic University of Pusan. 2012.

Lee JH, Kim SY. Comparative Effectiveness of Schroth Therapeutic Exercise Versus Sling Therapeutic Exercise in Flexibility, Balance, Spine Angle and Chest Expansion in Patient with Scoliosis. J Korean Soc Phys Med. 2014;9(1):11-23.

Lee SC, Kim TH, Cynn HS, et al. The Influence of Unstability of Supporting Surface on Trunk and Lower Extremity Muscle Activities During Bridging Exercise Combined With Core-Stabilization Exercise. Phys Ther Korea. 2010;17(1):17-25.

Lee YJ, Shim SS. The Effect of Core Muscle Strengthening Training on 1RM, Flexibility, Isokinetic Muscle Strength and Athletic Performance of Finswimming Players. The Korean Society Of Sports Science. 2012;21(5):961-71.

Marshall PW, Murphy BA. Core stability exercise on and off a Swiss ball. Arch Phys Med Rehabil. 2005:86(2):242-9.

Mika A, Clark BC, Oleksy Ł. The influence of high and low heeled shoes on EMG timing characteristics of the lumbar and hip extensor complex during trunk forward flexion and return task. Man Ther. 2013;18(6):506-11.

Miyake Y, Kobayashi R, Kelepecz D, et al. core exercises elevate trunk stability to facilitate skilled motor behavior of the upper extremities. J Bodyw Mov Ther. 2013;17(2):259-65.

O'Sullivan SB, Schmitz TJ. Physical Rehabilitation: Assesment and treatment $\left(4^{\mathrm{TH}}\right.$ ed). USA. F. A. Davis Company. 2001.

Park HJ, Oh DW, Kim SY. Effects of integrating hip movements into bridge exercises on electromyographic activities of selected trunk muscles in healthy individuals. Man Ther. 2014;19(3):246-51.

Park KJ. The effects of the iliopsoas muscle stretching and strengthening exercise on the static flexibility and pain intensity for the chronic low back pain patients Korean Acad Phys Ther Sci. 2009;16(1):11-20. 
Roh HL, Ma SY. Proprioceptive Motor Control on Trunk and Lower Extremity Muscle Activity. The Korea Contents Association. 2012;365-6.

Sekendiz B, Altun O, Korkusuz F, et al. Effects of Pilates exercise on trunk strength, endurance and flexibility in sedentary adult females. J Bodyw Mov Ther. 2007;11(4):318-326.

Seo DY, Lee YS, Lee DJ et al. The Effect of a Six-Week Sling Exercise on Flexibility, Balance, Muscular Strength, Pelvic Tilt Angle in Age of 20 Woman Low Back Pain Journal of Korean Society of
Integrative Medicine. 2013;1(2):1-12.

Son HH. Trunk Muscle Activation during Bridge Exercise with Various Shoulder Supporting Surfaces. J Korean Soc Phys Med. 2015;10(3):299-304.

Stevenson VK, Coorevits PL, Bouche KG, et al. The influence of specific training on trunk muscle recruitment patterns in healthy subjects during stabilization exercises. Man Ther. 2007;12(3):271-9.

Vera-Garcia FJ, Grenier SG, McGill SM. Abdominal muscle response during curl-ups on both stable and labile surfaces. Phys Ther. 2000;80(6):564-9. 